\title{
Signs and Symptoms of First-Onset TMD and Sociodemographic Predictors of Its Development: The OPPERA Prospective Cohort Study
}

\author{
Gary D. Slade ${ }^{\star}, \dagger, \ddagger$ Eric Bair ${ }^{\ddagger}, \S$, Joel D. Greenspan ${ }^{\#}$, Ron Dubner ${ }^{\#}$, Roger B. Fillingim ${ }^{\star *}$, \\ Luda Diatchenko ${ }^{\ddagger}$, , William Maixner ${ }^{\ddagger}, \S$, Charles Knott ${ }^{\dagger \dagger}$, and Richard Ohrbach ${ }^{\ddagger \ddagger}$ \\ *Department of Dental Ecology, University of North Carolina at Chapel Hill, Chapel Hill, North \\ Carolina ${ }^{\dagger}$ Department of Epidemiology, University of North Carolina at Chapel Hill, Chapel Hill, \\ North Carolina §Department of Endodontics, University of North Carolina at Chapel Hill, Chapel \\ Hill, North Carolina IDepartment of Biostatistics, University of North Carolina at Chapel Hill, \\ Chapel Hill, North Carolina ₹Regional Center for Neurosensory Disorders, University of North \\ Carolina at Chapel Hill, Chapel Hill, North Carolina \#Department of Neural and Pain Sciences, \\ and Brotman Facial Pain Center, University of Maryland Dental School of Dentistry, Baltimore, \\ Maryland ${ }^{*}$ University of Florida, College of Dentistry, and Pain Research and Intervention Center \\ of Excellence, Gainesville, Florida ${ }^{\dagger \dagger}$ Battelle Memorial Institute, Durham, North Carolina \\ ¥¥Department of Oral Diagnostic Sciences, University at Buffalo, Buffalo, New York
}

\section{Abstract}

\begin{abstract}
Although cross-sectional studies of temporomandibular disorders (TMDs) often report elevated prevalence in young women, they do not address the risk of its development. Here we evaluate sociodemographic predictors of TMD incidence in a community-based prospective cohort study of U.S. adults. Symptoms and pain-related disability in TMD cases are also described. People aged 18 to 44 years with no history of TMD were enrolled at 4 study sites when they completed questionnaires about sociodemographic characteristics. During the median 2.8-year follow-up period, 2,737 participants completed quarterly screening questionnaires. Those reporting symptoms were examined clinically and 260 had first-onset TMD. Additional questionnaires asked about severity and impact of their symptoms. Univariate and multivariate Cox regression models quantified associations between sociodemographic characteristics and TMD incidence. First-onset TMD developed in $3.9 \%$ of participants per annum, typically producing mild to moderate levels of pain and disability in cases. TMD incidence was positively associated with age, whereas females had only slightly greater incidence than males. Compared to whites, Asians had lower TMD incidence whereas African Americans had greater incidence, although the latter was attenuated somewhat after adjusting for satisfaction with socioeconomic circumstances.
\end{abstract}

Perspective-In this study of 18- to 44-year-olds, TMD developed at a higher rate than reported previously for similar age groups. TMD incidence was positively associated with age but weakly associated with gender, thereby differing from demographic patterns of prevalence found in some cross-sectional studies. Experiences related to aging merit investigation as etiologic influences on development of TMD.

(C) 2013 by the American Pain Society

Address reprint requests to Gary D. Slade, BDSc, DDPH, PhD, Department of Dental Ecology, Room 4501E, Koury Oral Health Sciences, UNC School of Dentistry, CB \#7455, Chapel Hill, NC 27599-7450. gary_slade@ dentistry.unc.edu.

Other authors declare no competing interests. 


\section{Keywords}

Temporomandibular joint disorders; prospective cohort studies; demography; socioeconomic factors; population characteristics

Evidence about demographic variation in temporomandibular disorders (TMDs) comes predominantly from cross-sectional studies of its prevalence. The findings are surprisingly varied, even for fundamental characteristics of age and gender. A systematic review of studies published before $1999^{22}$ concluded that prevalence was greater in women than in men, and that there was a negative association between age and TMD prevalence. However, in nationally representative, cross-sectional surveys of U.S. adults, prevalence in women resembled an inverted-U relationship, peaking around the fifth decade of life and, in some ages, did not differ markedly from prevalence in men. ${ }^{14,25,33}$

Because prevalence represents only a snapshot of illness within a population at a single point in time, cross-sectional studies do not address the risk of developing a condition over time. Instead, prospective cohort studies of incidence are needed. Furthermore, when the illness has a lengthy natural history, cross-sectional health surveys typically have a preponderance of chronic cases. Expressed arithmetically, prevalence of a disease in a fixed population is the product of its incidence and duration. ${ }^{29}$ It follows that prevalence and incidence of a chronic condition such as TMD might reveal markedly different demographic patterns.

Evidence about TMD incidence in adults comes from 3 population-based prospective cohort studies of TMD symptoms and 1 of TMD signs. In a 2-year follow-up of adults aged $\geq 18$ years in Seattle, WA, TMD incidence of symptoms was greater in females compared to males and it decreased in successively older age groups, although the youngest age category reported was 18 to 44 years. ${ }^{35}$ Similar associations of TMD symptoms with gender and age were reported in a 2-year prospective cohort study of UK adults aged 18 to 75 years in 2003 to 2004. ${ }^{1}$ Another U.S. study of 19-to 23-year-old women in California reported 3-fold greater incidence of TMD symptoms in Caucasians compared to African Americans. ${ }^{26}$ All 3 studies used symptoms of TMD reported in a single follow-up questionnaire to quantify incidence. At the 5-year follow-up examination in a prospective cohort study of a representative sample of adults living in Pomerania, Germany, incidence of TMD signs was assessed by palpation of masticatory muscles and joints. ${ }^{18}$ Age and gender were used as covariates in statistical models, but the direction or magnitude of their effects were not reported.

Another prominent feature of prospective cohort studies is that they measure putative risk factors prior to onset of illness, thus fulfilling a cardinal requirement that a causal influence must be present prior to onset of illness. ${ }^{12}$ Although this does not affect inferences about immutable demographic characteristics (ie, birth date, gender, race, and ethnicity), it can bias associations with socioeconomic characteristics that may change as a consequence of illness. For example, chronic, disabling pain may cause work loss, reducing income, thereby representing reverse causation in the association of socioeconomic status with pain. Evidence obtained from prospective cohort studies about mutable risk factors therefore is given greater weight than evidence obtained from cross-sectional study designs. ${ }^{5}$

These characteristics of TMD and its likely risk factors provided the motivation for the OPPERA (Orofacial Pain: Prospective Evaluation and Risk Assessment) project to undertake a prospective cohort study to investigate influences of a broad range of putative genetic, physiologic, psychosocial, and clinical risk factors on development of first-onset 
TMD. Many of those putative risk factors might, themselves, be dependent upon or modified by people's demographic and socioeconomic circumstances. The primary aim of this paper was to evaluate associations between sociodemographic characteristics and incidence of first-onset TMD in the OPPERA cohort of generally healthy, communitydwelling volunteers who did not have TMD when enrolled into the study. A secondary aim was to characterize signs and symptoms of TMD in the people who developed the condition.

\section{Methods}

This section summarizes study methods that are explained fully in the supplementary materials and elsewhere in this volume. ${ }^{3}$ Study participants provided informed, signed consent to participate in the study. The OPPERA project was reviewed and approved by institutional review boards at each of 4 study sites and at the data coordinating center, Battelle Memorial Institute.

\section{Study Design, Setting, and Participants}

This paper reports findings from the OPPERA prospective cohort study of 2,737 people who were enrolled in 2006 to 2008 and observed for up to 5.2 years, during which time 260 people developed TMD. The target population was adults aged 18 to 44 years with no significant history of TMD. Community-based volunteers were recruited at 4 U.S. study sites and examined using OPPERA's adaptation of a restricted version of the Research Diagnostic Criteria for TMD (RDC/TMD) to exclude people who had TMD. ${ }^{7}$ Furthermore, enrollees reported no orofacial pain in the month before enrollment and, prior to that period, no more than 4 days of orofacial pain per month. At enrollment, study participants also completed questionnaires, their autonomic function and sensitivity to sensory stimuli was measured, and a blood sample was collected for genotyping.

\section{Variables and Data Sources for This Analysis}

Questions used to evaluate sociodemographic characteristics at baseline have been described in detail elsewhere. ${ }^{33}$ In summary, study participants reported demographic details during a screening interview conducted by telephone. Age was reported in years, gender was reported as male or female, and 1 or more categories of race and ethnicity were endorsed. For this analysis, people were given a single classification of race/ethnicity according to the following criteria: 1) people reporting Hispanic ethnicity were classified as Hispanic, regardless of their reported race; 2) otherwise, if a single racial group of either white, black/ African American, or Asian was reported, the person was classified as such; 3) all other people were classified as "other race, multiple races or unstated."

During the baseline clinic visit at the time of study enrollment, study participants reported additional demographic and socioeconomic characteristics in a self-completed questionnaire. Lifetime residence in the United States was reported as yes or no, as was current health insurance coverage. First language spoken at home was classified as English or not English. Two groups were formed based on reported marital status: 1) never married; 2) married or cohabiting; and 3) divorced, separated, or widowed. Income and education were regarded as objective measures of socioeconomic status. Highest level of educational attainment was reported at 7 levels and classified into 4 groups for reporting purposes: 1) high school or less; 2) some college; 3) college graduate; or 4) postgraduate. Likewise, annual household income from all sources was reported at 7 levels and classified into 4 groups: 1) SUSD $20,000,2)$ USD 20,000 to $39,999,3)$ USD 40,000 to 79,999 , or 4) 2 USD 80,000 . Subjective assessments of socioeconomic status were based on reported satisfaction with financial circumstances and with material standards of life. Each was evaluated using an 11-point rating scale anchored at $0=$ totally dissatisfied and $10=$ totally satisfied. These measures of 
satisfaction with socioeconomic circumstances represent markers of financial and economic strain. ${ }^{19}$

At 3-monthly intervals after enrollment, study participants were asked to complete a screening questionnaire that asked about TMD pain symptoms. As described in detail elsewhere in the volume, ${ }^{3}$ those reporting TMD-related pain symptoms, together with a random sample of symptom-free people, were invited to research clinics for a follow-up examination that determined presence or absence of painful TMD. The threshold for pain symptoms was $\geq 5$ days for at least 1 of the preceding 3 months, including $\geq 1$ day in the preceding week. To address this paper's second aim, descriptive statistics were generated to characterize symptoms and signs of TMD. Symptoms in the 3 months prior to onset of TMD were reported in the quarterly screening questionnaire (QHU; see Appendix A of the supplementary material). Study participants were first asked if they had experienced "headaches or pain in your face, jaw, temples, in front of the ear, or in the ear." Those responding affirmatively were asked about the duration of pain, whether or not they had experienced other symptoms such as jaw stiffness or cramping, and whether or not a health care provider had diagnosed TMD. A few days before the clinic visit, symptomatic study participants were asked to completed the Multidimensional Pain Inventory, ${ }^{17}$ version 3, and the impact of pain was assessed using the Graded Chronic Pain Scale (GCPS) with a 6month reference period. ${ }^{36}$ At the clinic visit, facial pain during the preceding month was rated using 0 to 100 numeric ratings scales, and Gracely scales evaluated unpleasantness and intensity of facial pain. ${ }^{10}$

The definitive classification of first-onset TMD in 260 people required that they satisfy 2 criteria determined during follow-up examinations: 1) pain experienced for $\geq 5$ of the preceding 30 days within anatomic locations identified by the examiner and 2) examiner findings of pain in muscles (myalgia), joints (arthralgia), or both evoked by jaw maneuver and digital palpation of masticatory structures. Because examiners served as the reference ("gold") standard to classify TMD onset, it was possible that study participants who reported no pain in self-completed questionnaires could, nevertheless, be found to have pain in anatomic locations that were consistent with TMD as defined in this study. Examiners recorded the degree of jaw mobility and occurrence of evoked pain in the 2 temporomandibular joints and 8 groups of masticatory muscles: temporalis, masseter, submandibular and lateral pterygoid area, and posterior and submandibular, each assessed bilaterally. Pain was also recorded in response to digital palpation of 14 neck locations and 14 body locations.

\section{Statistical Methods}

Scores summarizing symptoms of TMD were computed for the 260 people with first-onset TMD using algorithms described previously. ${ }^{24}$ Rasch scores for 9 primary scales of the MPIv3 were computed with proprietary software ${ }^{1}$ and mean values for OPPERA participants were compared with corresponding scales reported for 6,532 people described as a "heterogeneous pain sample." 30 For descriptive purposes, the average annual incidence of first-onset TMD was calculated as the number of people with first-onset TMD divided by the sum of follow-up periods for all people who completed at least $1 \mathrm{QHU}$ screening questionnaire.

To test hypotheses about associations between baseline characteristics and TMD incidence, univariate hazard ratios were first computed using Cox proportional hazards regression. When the baseline risk factor was categorical, one category was nominated as the referent and dummy variables represented each of the other categories. Hazard ratios were computed both with adjustment for study site and with additional adjustment for demographics (age, gender, race/ethnicity, and lifetime U.S. residence). Hazard ratios were also computed using 
multiple imputation to account for 318 people who were not examined as intended for 1 of 2 reasons: 1) 243 people reported TMD symptoms in quarterly screening questionnaires but did not attend research centers for definitive examination; and 2) one examiner, who conducted 75 examinations, had a higher-than-expected rate of TMD. The method of multiple imputation is summarized in supplementary materials and described in detail elsewhere. ${ }^{3,6}$

Two strategies of multivariable modeling were used to evaluate combined effects of baseline characteristics on rate of first-onset TMD. The first strategy used multivariable Cox regression models to generate hazard ratios quantifying contributions of 4 core demographic variables (age, gender, race/ethnicity, and lifetime U.S. residence) to TMD incidence. Other socioeconomic variables found to be significant predictors of TMD incidence in the univariate analysis were then added to the model. The focus was on the change in magnitude of demographic hazard ratios, with the goal to assess the extent to which demographic associations with TMD incidence were confounded by socioeconomic characteristics. The degree of confounding was quantified as the percentage change in the hazard ratio after addition of putative confounding variables to the model. Comparable Poisson regression models were used to generate adjusted estimates of TMD incidence rates that were plotted for descriptive purposes.

The second strategy used random forest modeling ${ }^{13}$ to analyze potential contributions of all variables, not merely the reduced set used in the multivariable Cox model. The random forest model was created by generating a set of decision trees. A decision tree predicts an outcome by recursively partitioning the set of predictor variables producing results that can be visualized as a tree diagram. ${ }^{4}$ The number of predictors in each tree was chosen to be the square root of the number of predictors, which is a conventional approach used in random forest modeling.

This novel method of data mining was used to achieve 2 goals: 1) to identify the most important risk factors for first-onset TMD and 2) to generate plots depicting adjusted association between each variable and TMD incidence, with adjustment for the effects of other variables and with latitude in generating the plots that permitted departure from a straight-line association. The model produced importance scores, 1 for each variable, representing the decrease in the predictive accuracy of the model when the variable is measured incorrectly. This approach assigns the most important variable a score of 100, and all other importance scores have lower values that could range to a negative value if the variable worsened prediction. The random forest model was used also to compute the expected rate of first-onset TMD that would be observed at several values of the variable after averaging over the values of all other variables in the model. Partial dependence plots were then generated and loess smoothing was used to help visualize the association. ${ }^{21}$ The loess smooth was fit using the "lowess" function in R using the default parameters, which specifies that two-thirds of the points influence the smooth at each value.

The 2 strategies were selected in favor of other approaches for multivariable analysis for several reasons. The first strategy is a conventional approach that adjusts for potential confounding effects of variables identified a priori, based on conceptual relevance and univariate association with TMD incidence. However, it does not take advantage of information about the excluded variables. Thus, a random forest model was used to evaluate contributions of all variables. Random forests have several other advantages compared to conventional linear regression models. Specifically, random forests can impute for missing data and handle large numbers of correlated predictor variables without decreasing the accuracy of the model. ${ }^{11}$ 


\section{Results}

At enrollment, $85 \%$ of participants $(n=2,770)$ reported having never experienced orofacial pain, and the remaining $15 \%(\mathrm{n}=488)$ reported some history of orofacial pain that was below the enrollment-exclusion threshold of $\geq 5$ days per month. Of the 3,258 enrollees, 2,737 participants completed at least 1 follow-up questionnaire representing 7,404 personyears of follow-up (median $=2.8$ years per person), during which time 260 people developed first-onset TMD. This yielded an average annual TMD incidence rate of 3.5\% ( $95 \%$ confidence interval $=3.2 \%-3.9 \%$ ) of people per annum based on complete-case analysis. Imputation for 521 people lost to follow-up produced an incidence rate that was identical to 1 decimal place. However, the rate increased to $3.9 \%$ (95\% confidence interval $=$ $3.5 \%-4.3 \%$ ) after imputation for 318 people who were not examined as intended. As reported elsewhere, ${ }^{3}$ the imputed annual incidence rates at the 4 study sites were $1.8 \%$ (Chapel Hill, NC), 4.2\% (Gainesville, FL), 4.9\% (Buffalo, NY), and 5.3\% (Baltimore, MD).

\section{Characteristics of TMD in 260 Incident Cases}

In the 3 months prior to follow-up examinations in which TMD onset was determined, twothirds of people with first-onset TMD described having experienced "recurrent bouts" of facial pain or headache, whereas one-fifth described "persistent" pain (Table 1). Pain usually had lasted for either 1 to 4 days ( $40.4 \%$ of people) or 5 to 7 days ( $38.1 \%$ of people) out of the preceding 2 weeks, and painful jaw symptoms were described most frequently in terms of an ache, soreness, or tenderness. Stiffness or fatigue was the most common nonpainful jaw symptom. At the time of their clinic visit, $18.8 \%$ of people with first-onset TMD had no pain according to the Graded Chronic Pain Scale whereas $43.5 \%$ were classified with pain of low disability and low intensity (Grade I). Nearly one-quarter (23.3\%) of people characterized their facial pain as "headache only" (Table 1), which was similar to the percentage (18.9\%) that characterized their facial pain as "face or jaw only."

Using Gracely scales, the median rating of facial pain unpleasantness in TMD cases was consistent with the descriptor "slightly annoying" whereas median intensity fell between descriptions of "very mild" and "mild" (Table 2). Median ratings tended to be in the lower half of scales used to quantify degree of pain. For example, during the preceding 30 days, the median rating of average pain was 30 on the 0 to 100 scale, and during the preceding 6 months, it was 4 on the 0 to 10 scale. One half of people with first-onset TMD reported no interference with work or social activities. Average scores for most of MPIv3 scales showed less impact from TMD pain in these cases with first-onset TMD compared to the impact experienced by a heterogeneous chronic pain sample (Fig 1).

During examination, 60 TMD cases were classified as myalgia alone, 10 as arthralgia alone and 190 as both myalgia and arthralgia. Median values for pain during palpation and jaw movement were all in the lower half of the possible range (Table 3). For example, pain was elicited at a median of 2 muscle groups from among the 8 muscle groups evaluated during jaw opening. During palpation, the masseter was the most likely to elicit pain (medians of 5 sites in the right masseter and 4 sites in the right masseter from among 10 sites evaluated in each) whereas the submandibular and lateral pterygoid muscles were least likely to elicit palpation-evoked pain. Palpation elsewhere in the body elicited pain in a median of 3 sites in the neck (from among 14 sites evaluated) and at a median of 4 sites in the body (from among 14 sites evaluated).

For the complete cohort of 2,737 people, the site-adjusted incidence rate of first-onset TMD increased according to age, from $2.5 \%$ per annum among 18 - to 24 -year-olds to $4.5 \%$ per annum among 35- to 44-year-olds (Table 4). Relative to 18- to 24-year-olds, the corresponding hazard ratios of 1.38 for 25- to 34 -year-olds and 1.46 for 35 - to 44 -year-olds 
were statistically significant after additional adjustment for other demographic characteristics and after imputation for people who were not examined as intended. When age was modeled as a continuous variable, the fully adjusted and imputed hazard ratio associated with an increase of 10 years in age was $1.21(95 \% \mathrm{CI}=1.02,1.43$; data not tabulated). When age-squared was added to the model, the effect was not statistically significant $(P=-.499)$, confirming that association with the continuous measure of age was linear.

Females had only marginally greater TMD incidence than males, and the fully adjusted and imputed hazard ratio of 1.30 was of borderline statistical significance $(P=.051$, Table 4$)$. Relative to whites, African Americans had significantly greater incidence of TMD, whereas Asians had significantly lower incidence, although the latter effect was attenuated $(P=.24)$ in the fully adjusted and imputed analysis. People who had lived for some period outside the United States had less than one half the incidence of TMD relative to lifetime U.S. residents, whereas language first spoken and marital status were not significantly associated with TMD incidence.

When interactions between pairs of the 4 main demographic characteristics were evaluated in Cox models, there was some evidence of interaction between age and race/ethnicity $(P=$. 055). Rates from a corresponding Poisson regression model showed only small age variation in incidence among whites, but pronounced age variation in incidence was observed among racial minorities (Fig 2A). Other interaction terms were not at all significant statistically: for age $\times$ gender, $P=.79$; for age $\times$ lifetime U.S. residence, $P=.88$; for gender $\times$ race/ ethnicity, $P=.76$; for gender $\times$ lifetime U.S. residence, $P=.95$; and for race $\times$ lifetime U.S. residence, $P=.25$. Age-and gender-specific rates from models with interactions of age $\times$ gender (Fig 2B) and gender $\times$ race (Fig 2C) are included in for comparison with crosssectional studies of TMD that have reported interaction in prevalence. ${ }^{14,33}$

Most socioeconomic measures were not significantly associated with TMD incidence (Table 5). The exception was the self-reported rating of satisfaction with material standards of life (hereafter "material satisfaction"), where the one-quarter of people with the lowest ratings had greater TMD incidence than the one-quarter of people with highest ratings. When the 0 to 10 numeric rating was used as a continuous variable, the fully adjusted and imputed hazard ratio was $.87(95 \% \mathrm{CI}=-.76, .98)$ for the effect of an increase of 1 standard deviation in material satisfaction (data not tabulated).

A multivariable Cox regression model that evaluated combined effects of all 4 demographic characteristics and material satisfaction revealed that TMD incidence was associated with greater age (modeled as a continuous variable), female gender, black/African American race, lifetime U.S. residence, and lower material satisfaction (Table 6). Hazard ratios were not markedly attenuated compared to results that adjusted only for demographics, reported above. The largest change was observed from African American race, where the hazard ratio reduced by $6 \%$, from 1.36 in the demographically adjusted model (Table 5) to 1.27 with adjustment for material satisfaction (Table 6). This represents only minimal confounding of the race/ethnicity association with TMD incidence.

In the random forest model that predicted incidence using all sociodemographic variables reported in Tables 4 and 5, the relationship between age and TMD incidence resembled a straight line (Fig 3A). However, there was a threshold effect in the inverse relationship between material satisfaction and TMD incidence (Fig 3B) in which satisfaction ratings of 7 or higher were not associated with TMD incidence. 


\section{Discussion}

First-onset TMD developed at a rate of nearly $4 \%$ of people per annum in this U.S. community-based cohort of 18- to 44-year-olds, usually producing mild to moderate levels of pain and disability. When examined, three-quarters of TMD cases had myalgia with arthralgia, and most reported pain when palpated at noncranial locations. Despite having examiner-classified TMD, one-quarter of cases described their pain as headache, not jaw pain. Greater age and lifetime U.S. residence were associated with increased incidence of TMD, whereas females had only slightly greater incidence than males. Compared to whites, African Americans had greater incidence and Asians had lower incidence of TMD, although the effect for African Americans was attenuated slightly after adjusting for material satisfaction. Objective measures of socioeconomic status did not predict TMD incidence.

Loss to follow-up is potentially a major source of bias in prospective cohort studies, although the degree of such bias in this study was not pronounced. In a separate paper, ${ }^{3}$ we report that the observed incidence rate of $3.5 \%$ per annum did not change appreciably after accounting for 521 people who completed no QHU questionnaires. As reported here, it increased to $3.9 \%$ per annum after imputation for 318 people who were not examined as intended. Imputation tended to attenuate associations for most sociodemographic predictors (ie, yielding hazard ratios that were closer to the null value of 1), although imputation generally did not alter the nominal threshold of $P<.05$ for statistical significance.

The imputed incidence rate of $4.0 \%$ per annum in young women (Fig 2B) was very similar to the rate of $3.8 \%$ per annum reported in the Californian study of up to 5-year incidence in 19- to 23 -year-old women. ${ }^{26}$ However, the overall rate of $4.0 \%$ per annum in OPPERA was nearly twice the annual rate reported for similar age groups in 2 previous prospective cohort studies of TMD symptoms conducted in Seattle ${ }^{35}$ and the United Kingdom, ${ }^{1}$ and it was more than 4 times the rate of developing TMD palpation tenderness in the German prospective cohort study. ${ }^{18}$ Although underlying rates of TMD likely differ among the 4 populations studied, there are other methodologic differences that might contribute to differences in reported incidence rates among these studies. OPPERA participants were volunteers recruited by advertisements at 4 U.S. study sites, whereas the other 3 studies used random sampling methods to select from defined populations: young women living in one Californian county, ${ }^{26}$ adults of all ages in a health maintenance organization in Seattle, ${ }^{35}$ and adult patients of all ages registered with a general medical practice in the northwest of England. ${ }^{1}$ At enrollment, the OPPERA study excluded people who had experienced orofacial pain symptoms for $\geq 5$ days per month, whereas some other studies excluded enrollees who had orofacial pain for $\geq 1$ day per month. The 2 U.S. studies and the UK study used symptom classifications for incidence that had a lower threshold of pain density than the OPPERA threshold of $\geq 5$ of the preceding 30 days. Likewise, the German study classified people as cases if they had 1 or more muscle sites that were tender to palpation, lower than the threshold used in OPPERA. Like the German study, we excluded people who had examiner-classified TMD at enrollment, and like the Californian and UK studies, we excluded people who had any significant history of TMD symptoms at enrollment. However, we did not exclude the $15 \%$ of enrollees who had experienced orofacial pain for $<5$ days per month. This probably contributed to greater incidence in OPPERA compared to the Seattle study, which excluded people with any lifetime history of TMD.

Other reasons for the comparatively high rate of TMD incidence in OPPERA likely include the frequency and nature of screening questions. The 3 previous studies conducted only a single follow-up assessment of each study participant, whereas OPPERA participants were screened quarterly. A single follow-up assessment cannot validly identify TMD onset in people whose pain stopped a few months before the follow-up assessment. Indeed, the 
majority of people with TMD experience intermittent episodes. For example, $65 \%$ of the first-onset TMD cases in this study described their pain as "recurrent bouts." Hence, prospective cohort studies that use a single follow-up assessment are prone to underenumerating incident cases of TMD.

Compared to previous studies, the current study also differed in the types of pain evaluated when screening for TMD, explicitly including reference to "headache" when asking about pain in the face and jaw. The inclusion appears vindicated by the finding that nearly onequarter of people with examiner-classified TMD described their pain as headache, not jaw pain. Furthermore, the OPPERA screening questionnaire had a sensitivity of $90 \%,{ }^{3}$ which meant that virtually all people in the cohort who truly had TMD were examined for a definitive case classification. In contrast, values of sensitivity below $50 \%$ have been observed ${ }^{15}$ for single-item questions of the type used in previous population-based cohort studies. These methodologic features suggest that the annual incidence rate of $4 \%$ observed in OPPERA is probably a more valid estimate of TMD risk than previous cohort studies.

Levels of pain and related disability in people who developed TMD were similar to values reported previously for incident cases of TMD. For example, one-quarter of OPPERA's incident cases had GCPS levels of II or more, very similar to $26.3 \%$ reported for incident cases in the U.S. cohort study. ${ }^{35}$ Not surprisingly, these OPPERA cases with first-onset TMD had less intense pain, on average, than people with chronic TMD. For example, GCPS levels of II or more were found among 50\% of chronic TMD cases in the OPPERA baseline case-control study. Other studies of chronic TMD in the United States, ${ }^{36}$ Germany, ${ }^{16}$ Saudi Arabia, ${ }^{2}$ Italy, and Israel ${ }^{23}$ found that at least $50 \%$ of people had GCPS categories of II or higher.

The positive association between age and TMD incidence was consistent with the ageassociated increase in odds of chronic TMD seen in the OPPERA baseline case-control study. ${ }^{33}$ However, other demographic patterns of association differed markedly between these 2 OPPERA studies. Females had substantially elevated odds of chronic TMD although their incidence of TMD was only slightly greater than males'. Racial/ethnic minorities had much lower odds of chronic TMD than whites, whereas African Americans had greater incidence than whites. Lifetime U.S. residence was not significantly associated with chronic TMD but was strongly associated with greater incidence of TMD. One explanation is that demographic factors might contribute differentially to onset of TMD versus persistence of symptoms that are the hallmark of chronic TMD. (The case classification for first-onset TMD used a shorter history of symptoms than the classification of chronic TMD in the OPPERA baseline case-control study that required a 6-month history of pain on $\geq 5$ days per month, including $\geq 15$ days in the month preceding the examination. ${ }^{33}$ ) Another possibility is that the case-control findings in OPPERA were an artifact of selection biases that have greater potential to affect case-control studies than prospective cohort studies. However, age and gender patterns seen in the baseline case-control study resembled findings from population benchmarks, ${ }^{33}$ suggesting that selection was not a serious problem in that study.

It is plausible that gender might contribute differentially to the onset and persistence of TMD, based on findings from one study of people with acute TMD which found that women were more likely than men to transition from acute to chronic TMD. ${ }^{8}$ Proposed reasons for gender differences in occurrence of chronic TMD include psychological characteristics that differ between men and women and potential biologic effects of female reproductive hormones. ${ }^{20}$ This study did not measure hormones and it was restricted to 18 - to 44 -yearolds, effectively excluding postmenopausal women, whose rates of TMD incidence might help address the question. Nonetheless, the current findings suggest that gender-related 
biological or psychological influences on risk of TMD might be critical in determining the transition from first-onset to chronic TMD.

Although the 2 OPPERA studies were consistent in finding a positive association between age and TMD incidence, the U.S. and UK prospective cohort studies of TMD found a negative association with age. 1,35 However, 3 caveats should be noted. The U.S. and UK studies reported wider age ranges than OPPERA, which might have masked age-related trends seen among 18-to 44-year-olds in those studies. Also, the 2 previous studies did not report racial/ethnic composition of their samples, and the positive age association in OPPERA was seen primarily in nonwhites (Fig 2). Likewise, in the U.S. population, the positive association between age and prevalence of TMD was more pronounced in racial minorities than in non-Hispanic whites. ${ }^{25}$ Finally, as noted above, OPPERA used different methods of follow-up and TMD case classification compared to previous studies that might have revealed different demographic patterns of association with TMD incidence. Another important consideration is that demographic characteristics are, to a large degree, proxy indicators of truly etiologic risk factors that contribute to TMD. For example, as reported elsewhere in this volume, the observed positive association between age and TMD incidence in this cohort was attenuated considerably after adjustment for measures of poor health status, which also was age-related. 31

It was surprising that TMD incidence was associated with a subjective measure of material satisfaction but not with objective measures of socioeconomic status, such as education and income. This might indicate that perceptions about socioeconomic status are more important in protecting against painful TMD than people's actual socioeconomic resources. However, little is known of potential mechanisms by which socioeconomic status might contribute to TMD development. The UK and German prospective cohort studies did not analyze the association ${ }^{1,18}$ whereas the U.S. study reported no association with educational attainment. ${ }^{35}$ Cross-sectional study designs report mixed findings, with many finding a nonsignificant association or nonlinear gradient between levels of socioeconomic status and occurrence of TMD. ${ }^{22}$ Hence, it is premature to speculate about pathways linking perceived social status and risk of TMD.

A novel finding was the very low incidence of TMD observed in people who had lived some of their lives outside the United States, an effect that persisted after adjustment for race/ ethnicity. This group comprises foreign-born immigrants to the United States and people born in the United States who had lived some time abroad, although there were no additional questions to make that distinction in this study. First-generation immigrants have better health outcomes, on average, than native-born residents for a variety of diseases and disorders, a phenomenon labeled the "healthy migrant effect."27,28 Meanwhile, relatively good health is probably a prerequisite for living abroad temporarily. As reported elsewhere, ${ }^{32}$ poor health was strongly associated with greater incidence of TMD incidence in this study. Hence, it seems possible that lower TMD incidence observed in people who were not lifetime residents of the United States might be attributable to their comparatively good general health.

Because age, gender, and race/ethnicity are fundamental to our understanding of TMD epidemiology, their influences merit further attention in future studies, including reporting age in small ranges, where possible. The current finding of heterogeneity in age associations among racial/ethnic groups should motivate more etiologically focused investigations. For example, social and economic disadvantage is thought to accelerate the development of agerelated diseases in African Americans more rapidly than in whites in a process termed "weathering." "In the U.S. population, biologic mediators of weathering were elevated in African Americans compared to whites ${ }^{9}$ and those same mediators were associated with 
some chronic pain conditions. ${ }^{34}$ However, those effects have yet to be investigated in studies of TMD. Another important caveat is that this study's finding of greater TMD incidence in African Americans compared to whites is in contrast to the one other study ${ }^{26}$ of TMD incidence to have compared the 2 groups.

In summary, first-onset TMD occurred at a greater rate in this cohort than in previous prospective cohort studies. This might be due to population differences in TMD incidence, although part of the reason probably relates to quarterly monitoring of TMD symptoms during follow-up in OPPERA. Associations with gender, race/ethnicity, and lifetime U.S. residence also contrast with patterns seen in OPPERA's baseline case-control study of chronic TMD, illustrating that sociodemographic characteristics can contribute differentially to first-onset TMD versus chronic TMD. Findings should not be extrapolated beyond the study population or the condition studied: this was a generally healthy cohort of 18 - to 44year-olds, and most participants who developed first-onset TMD had experienced symptoms for only 1 or 2 months. Nonetheless, the results challenge a widely held view that TMD is predominantly a condition of females in early adulthood, and they suggest that, even in early adulthood, ill health or other experiences related to aging could be important etiologic influences on risk of developing TMD.

\section{Acknowledgments}

This work was supported by NIH grant U01DE017018 and P01NS045685. This material was also supported with by the North Florida/South Georgia Veterans Health System, Gainesville, FL. The OPPERA program also acknowledges resources specifically provided for this project by the respective host universities: University at Buffalo, University of Florida, University of Maryland-Baltimore, and University of North Carolina- Chapel Hill. R.F. and G.S. are consultants and equity stock holders, and W.M. and L.D. are cofounders and equity stock holders in Algynomics, Inc., a company providing research services in personalized pain medication and diagnostics.

The authors would like to thank the OPPERA research staff for their invaluable contributions to this work. In addition, we express our gratitude to the participants who have devoted time and effort in support of this research. This work was done at the University of North Carolina at Chapel Hill; University at Buffalo, New York; University of Maryland-Baltimore; University of Florida, Florida; and Battelle Memorial Institute, North Carolina.

\section{References}

1. Aggarwal VR, Macfarlane GJ, Farragher TM, McBeth J. Risk factors for onset of chronic oro-facial pain-results of the North Cheshire oro-facial pain prospective population study. Pain. 2010; 149:354-359. [PubMed: 20304556]

2. Al-Harthy M, Al-Bishri A, Ekberg E, Nilner M. Temporomandibular disorder pain in adult Saudi Arabians referred for specialised dental treatment. Swed Dent J. 2010; 34:149-158. [PubMed: 21121414]

3. Bair E, Brownstein N, Ohrbach RO, Greenspan JD, Dubner R, Fillingim RB, Diatchenko L, Smith S, Maixner W, Gonzalez Y, Gordon S, Lim PF, Ribeiro-Dasilva M, Dampier D, Knott C, Slade GD. Study design, methods, sample characteristics and loss-to-follow-up: the OPPERA prospective cohort study. J Pain. in press (in this volume).

4. Breiman, L.; Friedman, J.; Olshen, R.; Stone, C. Classification and Regression Trees. New York: Wadsworth; 1984.

5. Briss PA, Zaza S, Pappaioanou M, Fielding J, Wright-De Aguero L, Truman BI, Hopkins DP, Mullen PD, Thompson RS, Woolf SH, Carande-Kulis VG, Anderson L, Hinman AR, McQueen DV, Teutsch SM, Harris JR. Developing an evidence-based Guide to Community Preventive Services—methods. The Task Force on Community Preventive Services. Am J Prev Med. 2000; 18:35-43. [PubMed: 10806978]

6. Brownstein, N.; Cai, J.; Slade, G.; Bair, E. [Accessed April 18, 2013] Parameter estimation in Cox proportional hazard models with missing censoring indicators. Available at: http://arxiv.org/abs/ 1304.3839 
7. Dworkin S, LeResche L. Research diagnostic criteria for temporomandibular disorders: Review, criteria, examinations and specifications, critique. J Craniomandib Disord. 1992; 6:301-355. [PubMed: 1298767]

8. Garofalo JP, Gatchel RJ, Wesley AL, Ellis E 3rd. Predicting chronicity in acute temporomandibular joint disorders using the research diagnostic criteria. J Am Dent Assoc. 1998; 129:438-447. [PubMed: 9573694]

9. Geronimus AT, Hicken M, Keene D, Bound J. "Weathering" and age patterns of allostatic load scores among blacks and whites in the United States. Am J Public Health. 2006; 96:826-833. [PubMed: 16380565]

10. Gracely RH, McGrath F, Dubner R. Ratio scales of sensory and affective verbal pain descriptors. Pain. 1978; 5:5-18. [PubMed: 673440]

11. Hastie, T.; Tibshirani, R.; Friedman, J. The Elements of Statistical Learning: Data Mining, Inference, and Prediction. New York: Springer; 2009.

12. Hill AB. The environment and disease: Association or causation? Proc R Soc Med. 1965; 58:295300. [PubMed: 14283879]

13. Ishwaran H, Kogalur UB, Blackstone EH, Lauer MS. Random survival forests. Ann Appl Stat. 2008; 2:841-860.

14. Isong U, Gansky S, Plesh O. Temporomandibular joint and muscle disorder-type pain in U.S. adults: The National Health Interview Survey. J Orofac Pain. 2008; 22:317-322. [PubMed: 19090404]

15. Janal M, Raphael K, Nayak S, Klausner J. Prevalence of myofascial temporomandibular disorder in US community women. J Oral Rehabil. 2008; 35:801-809. [PubMed: 18976276]

16. John MT, Reissmann DR, Schierz O, Wassell RW. Oral health-related quality of life in patients with temporomandibular disorders. J Orofac Pain. 2007; 21:46-54. [PubMed: 17312641]

17. Kerns RD, Turk DC, Rudy TE. The West Haven-Yale Multidimensional Pain Inventory (WHYMPI). Pain. 1985; 23:345-356. [PubMed: 4088697]

18. Kindler S, Samietz S, Houshmand M, Grabe HJ, Bernhardt O, Biffar R, Kocher T, Meyer G, Volzke H, Metelmann HR, Schwahn C. Depressive and anxiety symptoms as risk factors for temporomandibular joint pain: A prospective cohort study in the general population. J Pain. 2012; 13:1188-1197. [PubMed: 23141187]

19. Krause N, Baker E. Financial strain, economic values, and somatic symptoms in later life. Psychol Aging. 1992; 7:4-14. [PubMed: 1558704]

20. LeResche L. Epidemiology of temporomandibular disorders: Implications for the investigation of etiologic factors. Crit Rev Oral Biol Med. 1997; 8:291-305. [PubMed: 9260045]

21. Loader, C. Local Regression and Likelihood. New York: Springer; 1999.

22. Macfarlane TV, Glenny AM, Worthington HV. Systematic review of population-based epidemiological studies of orofacial pain. J Dent. 2001; 29:451-467. [PubMed: 11809323]

23. Manfredini D, Ahlberg J, Winocur E, Guarda-Nardini L, Lobbezoo F. Correlation of RDC/TMD axis I diagnoses and axis II pain-related disability. A multicenter study Clin Oral Investig. 2011; 15:749-756.

24. Ohrbach R, Fillingim RB, Mulkey F, Gonzalez Y, Gordon S, Gremillion H, Lim PF, RibeiroDasilva M, Greenspan JD, Knott C, Maixner W, Slade G. Clinical findings and pain symptoms as potential risk factors for chronic TMD: Descriptive data and empirically identified domains from the OPPERA case-control study. J Pain. 2011; 12:T27-T45. [PubMed: 22074750]

25. Plesh O, Adams SH, Gansky SA. Racial/ethnic and gender prevalences in reported common pains in a national sample. J Orofac Pain. 2011; 25:25-31. [PubMed: 21359234]

26. Plesh O, Gansky S, Curtis DO. Chronic pain in a biracial cohort of young women. Open Pain J. 2012; 5:24-31.

27. Razum O. Commentary: Of salmon and time travellers - musing on the mystery of migrant mortality. Int J Epidemiol. 2006; 35:919-921. [PubMed: 16847016]

28. Razum O, Zeeb H, Rohrmann S. The "healthy migrant effect"-Not merely a fallacy of inaccurate denominator figures. Int J Epidemiol. 2000; 29:191-192. [PubMed: 10750623] 
29. Rothman, KJ.; Greenland, S.; Lash, TL. Modern Epidemiology. 3rd. Philadelphia, PA: Wolters Kluwer Health/Lippincott Williams \& Wilkins; 2008.

30. Rudy, TE. [Accessed November 6, 2012] Multidimensional Pain Inventory Normative Data. Available at: http://www.pain.pitt.edu/mpi/MPI_Norms.pdf

31. Sanders AE, Ohrbach R. General health status and incidence of first-onset temporomandibular disorder: OPPERA prospective cohort study. J Pain. in press (in this volume).

32. Sanders AE, Slade GD, Bair E, Fillingim RB, Knott C, Dubner R, Greenspan JD, Maixner W, Ohrbach R. General health status and incidence of first-onset temporomandibular disorder: OPPERA prospective cohort study. J Pain. in press (in this volume).

33. Slade GD, Bair E, By K, Mulkey F, Baraian C, Rothwell R, Reynolds M, Miller V, Gonzalez Y, Gordon S, Ribeiro-Dasilva M, Lim PF, Greenspan JD, Dubner R, Fillingim RB, Diatchenko L, Maixner W, Dampier D, Knott C, Ohrbach R. Study methods, recruitment, sociodemographic findings, and demographic representativeness in the OPPERA study. J Pain. 2011; 12:T12-T26. [PubMed: 22074749]

34. Slade GD, Sanders AE, By K. Role of allostatic load in sociodemographic patterns of pain prevalence in the U.S. population. J Pain. 2012; 13:666-675. [PubMed: 22677453]

35. Von Korff M, Le Resche L, Dworkin SF. First onset of common pain symptoms: A prospective study of depression as a risk factor. Pain. 1993; 55:251-258. [PubMed: 8309712]

36. Von Korff M, Ormel J, Keefe FJ, Dworkin SF. Grading the severity of chronic pain. Pain. 1992; 50:133-149. [PubMed: 1408309] 


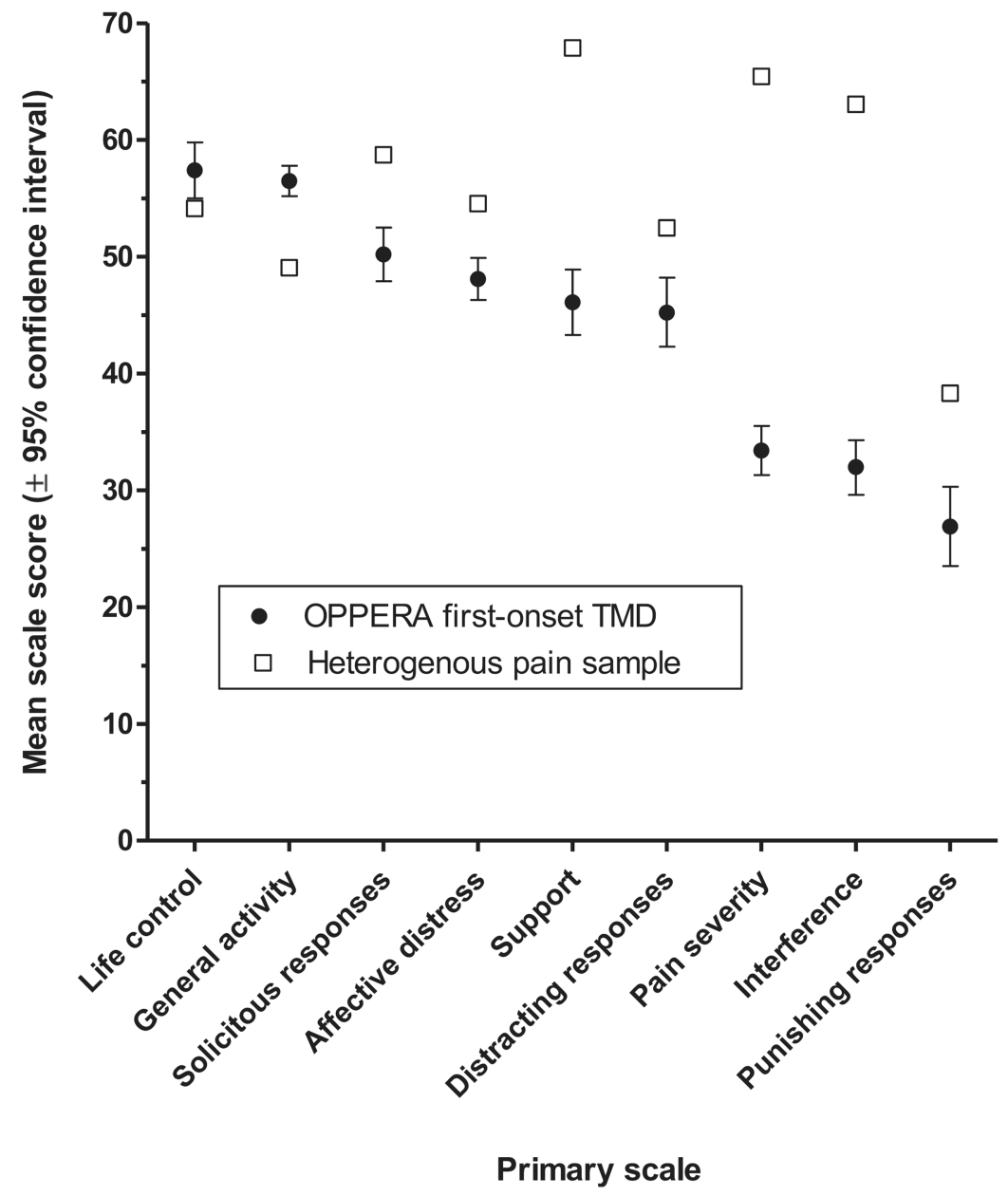

Figure 1.

Mean values for heterogeneous chronic pain sample $\left(n=6,532\right.$ people) are from Rudy. ${ }^{30}$ Error bars showing $95 \%$ confidence intervals for the heterogeneous pain sample are not visible because they are smaller than the boxes used as symbols. 
A Age $x$ Race/ethnicity interaction $\begin{array}{cccc}H R=1.43 & H R=2.65 & H R=1.50 & H R=0.82 \\ (1.09,1.87) & (1.08,6.50) & (0.52,4.34) & (0.32,2.07)\end{array}$

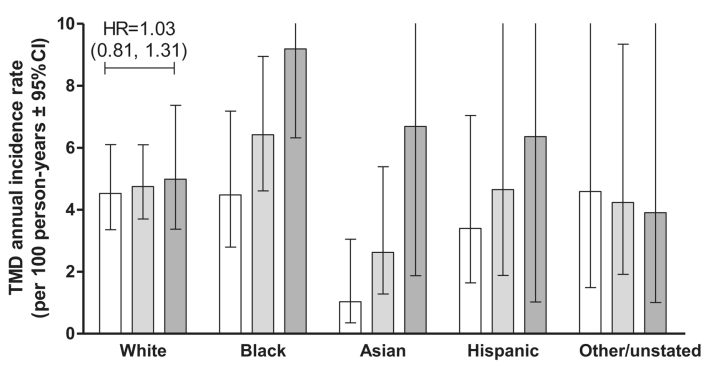

Age (years)

$\square 20 \square 30 \quad \square 40$

C Gender x Race interaction

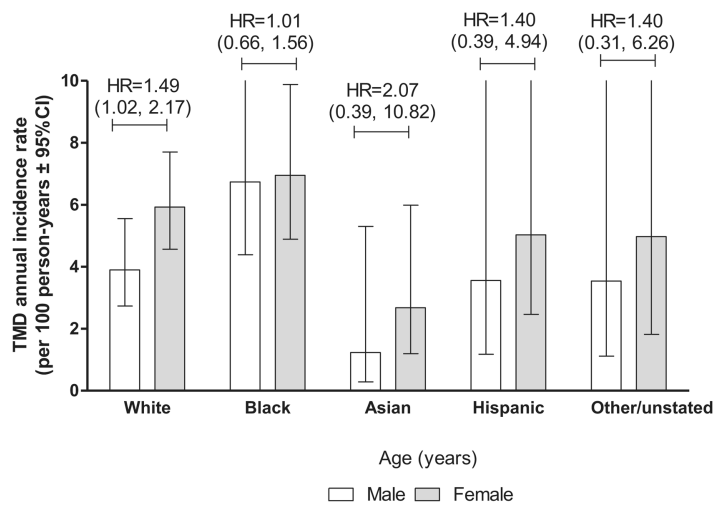

B Age $x$ gender interaction

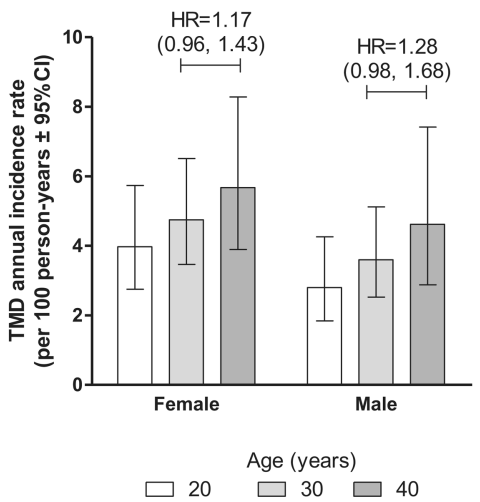

Figure 2.

Incidence rates of first-onset TMD were computed using multivariable Poisson regression models with multiple imputation to account for subjects who were not examined as intended. Covariates were study site (categorical variable, 4 levels), age (in years, with rates estimated for 3 selected age groups: 20, 30, and 40 years), race/ethnicity (5 categories), and lifetime U.S. residence ( 2 categories). The vertical axis has a maximum value of $10 \%$ per annum, truncating upper bounds of $95 \%$ confidence intervals for some rates. Hazard ratios (HRs) associated with 10-year difference in age (A and $\mathbf{B})$ or with female gender $(\mathbf{C})$ are shown with $95 \%$ confidence intervals in parentheses. They were calculated using multivariable Cox regression models with the same covariates described for the Poisson models. A includes an interaction term for age $\times$ race/ethnicity interaction $(P=.055)$. B includes an interaction term for age $\times$ gender interaction $(P=.79)$. $\mathbf{C}$ includes an interaction term for race $\times$ gender interaction $(P=.76)$. 

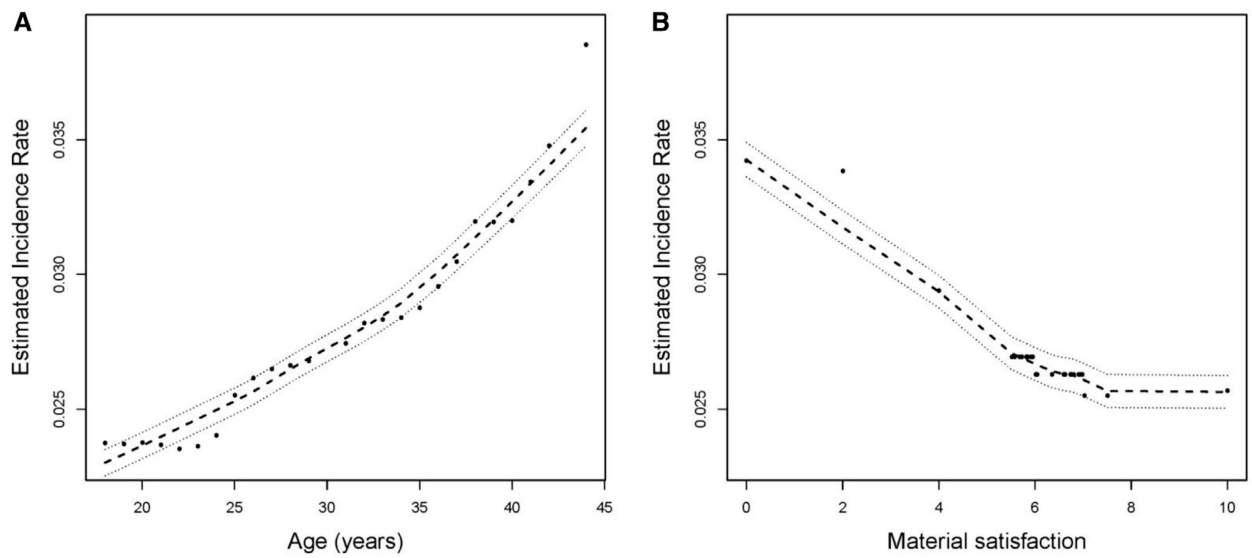

Figure 3.

TMD incidence rates, expressed as cases per 100 person-years, were generated from random forest models that predicted TMD onset using study site and sociodemographic variables reported in Tables 4 and 6 . Predicted values $(0)$ are plotted together with loess-smoothed estimates (- - ) and their $95 \%$ confidence intervals $(\cdots)$. Age was reported in years, and satisfaction with material standards of life was rated on a scale from 0 (totally dissatisfied) to 10 (totally satisfied). 
Table 1

Symptoms Related to TMD Reported by 260 People Who Developed First-Onset TMD: OPPERA Prospective Cohort Study, 2006 to 2011

\begin{tabular}{|c|c|c|}
\hline & Number of People & $\%$ \\
\hline \multicolumn{3}{|c|}{ Symptoms during the 3 months before clinical case classification ${ }^{*}$} \\
\hline \multicolumn{3}{|c|}{ Any headaches or facial pain } \\
\hline Yes & 253 & 97.3 \\
\hline No & 6 & 2.3 \\
\hline Not stated & 1 & .4 \\
\hline \multicolumn{3}{|c|}{ Duration of headaches or facial pain } \\
\hline None & 6 & 2.3 \\
\hline Persistent & 50 & 19.2 \\
\hline Recurrent bouts & 169 & 65.0 \\
\hline One time & 32 & 12.3 \\
\hline Not stated & 3 & 1.2 \\
\hline \multicolumn{3}{|c|}{ Number of months with $\geq 5$ consecutive days of facial pain } \\
\hline 0 & 18 & 6.9 \\
\hline 1 & 93 & 35.8 \\
\hline 2 & 90 & 34.6 \\
\hline$\geq 3$ & 58 & 22.3 \\
\hline Not stated & 1 & .4 \\
\hline \multicolumn{3}{|c|}{ Number of days with facial pain in last 2 weeks } \\
\hline None & 9 & 3.5 \\
\hline $1-4$ & 105 & 40.4 \\
\hline $5-7$ & 99 & 38.1 \\
\hline 28 & 46 & 17.7 \\
\hline Not stated & 1 & .4 \\
\hline \multicolumn{3}{|l|}{ Symptoms in face or jaw ${ }^{\dagger}$} \\
\hline Stiffness or tightness & 84 & 32.3 \\
\hline Cramping & 29 & 11.2 \\
\hline Fatigue or weakness & 33 & 12.7 \\
\hline Pressure & 67 & 25.8 \\
\hline Soreness or tenderness & 113 & 43.5 \\
\hline Ache or dull ache & 130 & 50.0 \\
\hline None of the above & 61 & 23.5 \\
\hline \multicolumn{3}{|c|}{ TMD diagnosed by health care provider } \\
\hline Yes & 10 & 3.9 \\
\hline No & 241 & 92.7 \\
\hline Not stated & 9 & 3.5 \\
\hline \multicolumn{3}{|c|}{ Symptoms reported on the day of clinical case-classification } \\
\hline \multicolumn{3}{|l|}{ Description of pain } \\
\hline Headache only & 58 & 23.3 \\
\hline
\end{tabular}




\begin{tabular}{|c|c|c|}
\hline & Number of People & $\%$ \\
\hline Pain in face, jaw, temple, in the ear, or in front of the ear & 47 & 18.9 \\
\hline Both headache and pain in face, etc & 130 & 52.2 \\
\hline None of the above & 14 & 5.6 \\
\hline Not stated & 8 & \\
\hline \multicolumn{3}{|l|}{ Facial GCPS level $\S$} \\
\hline 0: no disability & 49 & 18.8 \\
\hline I: low disability-low intensity & 113 & 43.5 \\
\hline IIa: low disability-high intensity & 26 & 10.0 \\
\hline IIb-IV: high disability with moderate/ severe limitation & 37 & 14.2 \\
\hline Not asked & 35 & 13.5 \\
\hline \multicolumn{3}{|l|}{ Reference period for symptoms was the preceding 3 months. } \\
\hline \multicolumn{3}{|l|}{${ }^{\dagger}$ Respondents were asked to endorse all symptoms that applied. } \\
\hline
\end{tabular}




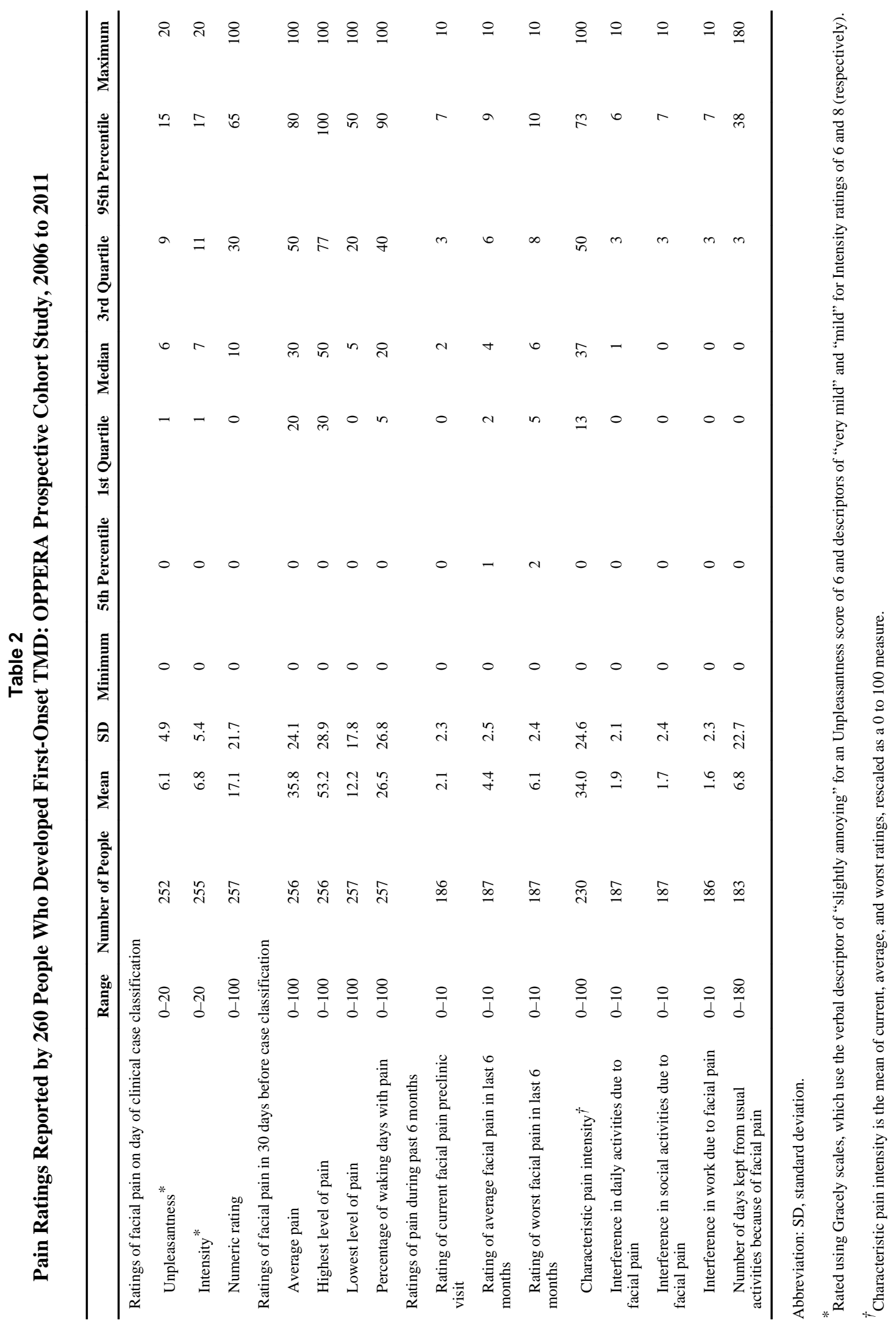

J Pain. Author manuscript; available in PMC 2014 December 01. 


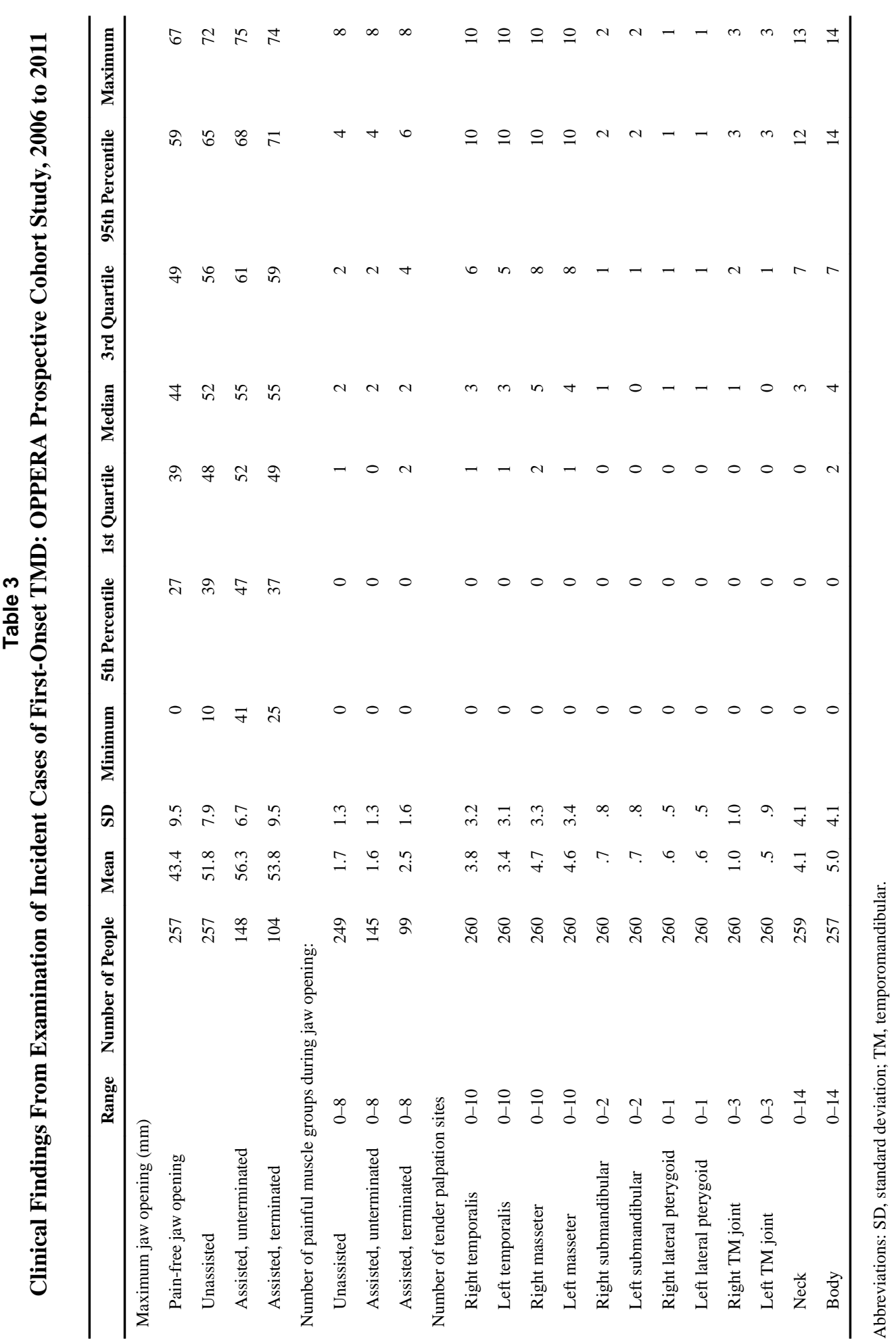

J Pain. Author manuscript; available in PMC 2014 December 01. 


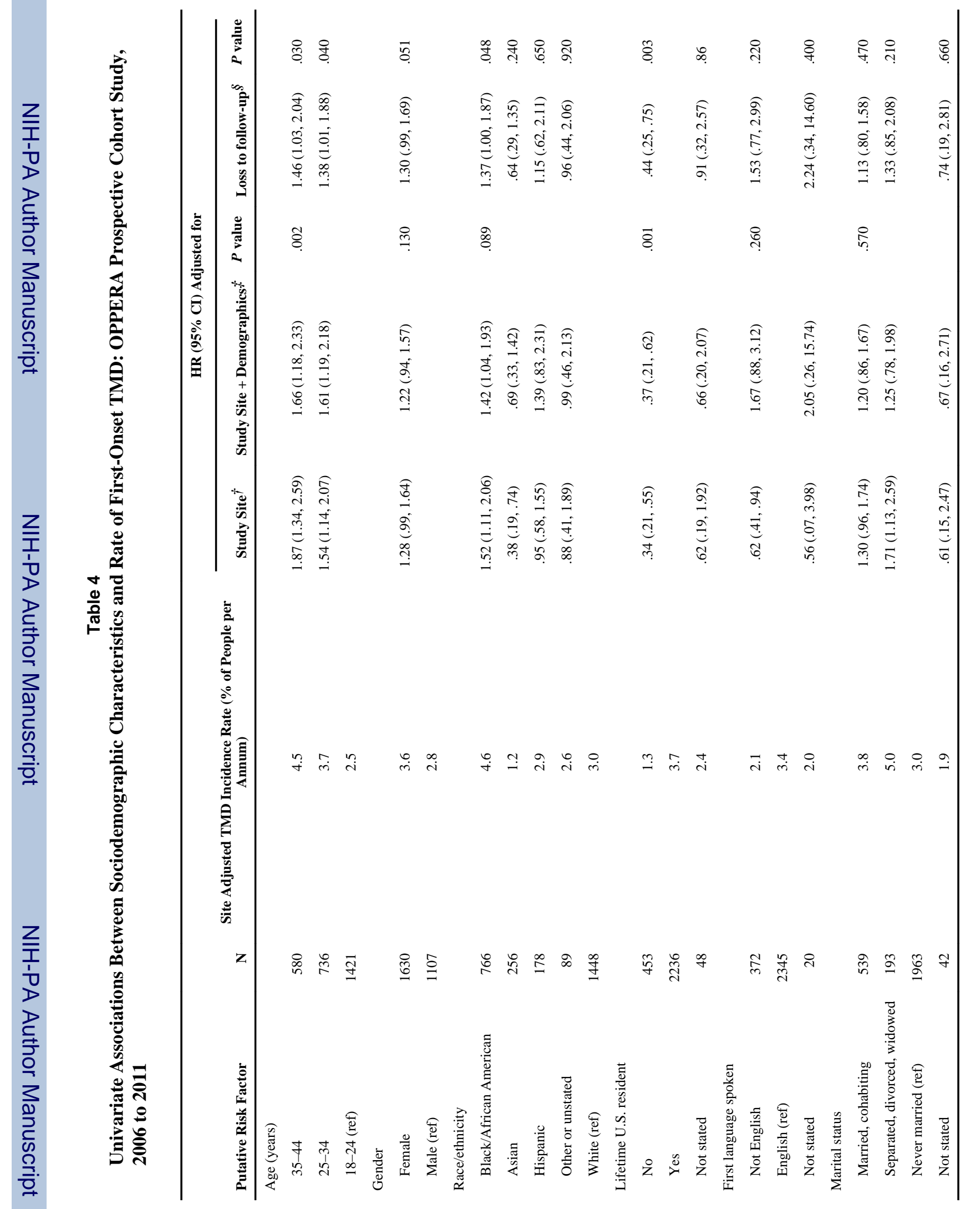

J Pain. Author manuscript; available in PMC 2014 December 01. 


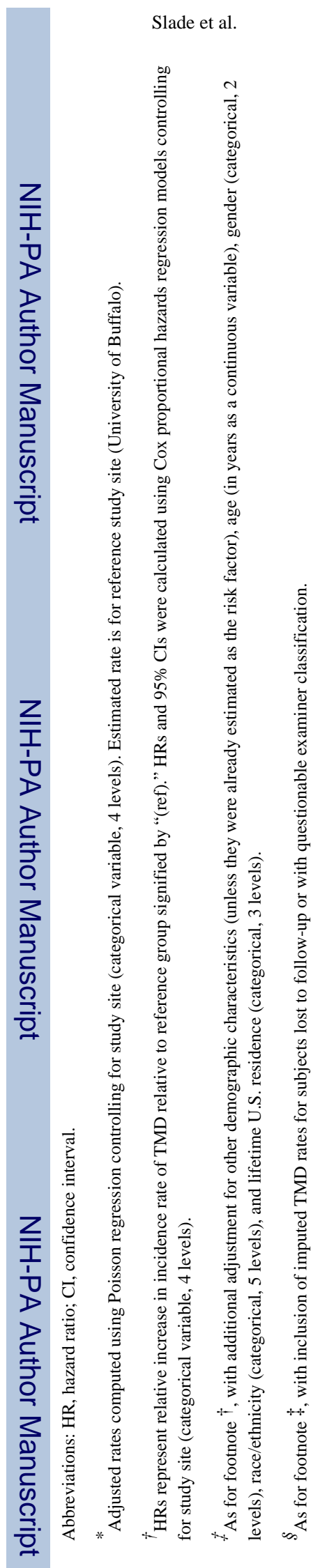

Page 22

J Pain. Author manuscript; available in PMC 2014 December 01. 


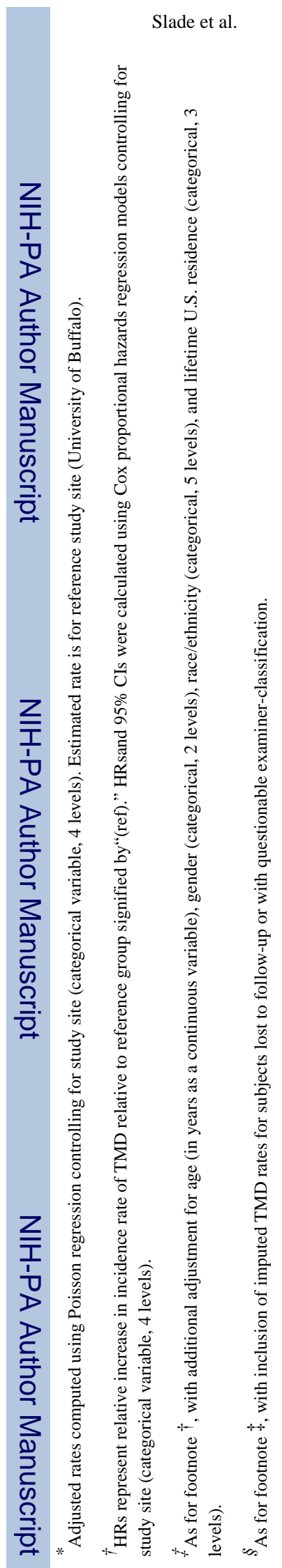

Page 24

J Pain. Author manuscript; available in PMC 2014 December 01. 
Table 6

Multivariable Associations of Sociodemographic Characteristics With TMD Incidence: OPPERA Prospective Cohort Study, 2006 to 2011

\begin{tabular}{llc}
\hline Predictor Variable & Coding & HR $(\mathbf{9 5 \%}$ CI $)$ \\
\hline Age & Decades & $1.18(1.00,1.40)$ \\
Gender $($ ref = male) & Female & $1.34(1.03,1.75)$ \\
Race $($ ref = white) & Black/African American & $1.27(.92,1.74)$ \\
& Asian & $.59(.28,1.26)$ \\
& Hispanic & $1.08(.59,1.98)$ \\
Lifetime residence (ref = yes) & Other & $.92(.43,1.98)$ \\
Rating of satisfaction with material standards of life $($ ref $=$ high $[9-10])$ & Low $(0-5)$ & $.49(.30, .81)$ \\
& Mid $(6-8)$ & $1.71(1.17,2.52)$ \\
& Not stated & $1.45(1.02,2.06)$ \\
\hline
\end{tabular}

Abbreviations: HR, hazard ratio; CI, confidence interval

NOTE. $95 \% \mathrm{CI}=95 \%$ confidence intervals computed using Cox proportional hazards regression models, additionally controlling for study site (categorical variable, 4 levels), with multiple imputation to account for subjects lost to follow-up or with questionable examiner classification. 